\title{
Expanding the responsibility of an offshore team and sustainably increasing business value using SAFe
}

\author{
Alaukikdev Pandya, V. S. Mani, Ansuman Pattanayak \\ Siemens Healthcare Pvt. Ltd. Bangalore, India \\ alaukikdev.pandya@siemens-healthineers.com,vs.mani@siemens-healthineers.com, ansuman.pattanayak@siemens-healthineers.com
}

\begin{abstract}
The paper outlines a four-year journey of a software engineering team based in Bangalore, India, which transitioned from having the responsibility of different modules to complete engineering responsibility of the range of software products. The paper also talks about the proactive steps taken to transition to Scaled Agile Framework (SAFe) [1] successfully and discusses the challenges related to people and team culture. The paper also summarizes lessons learned from the journey.
\end{abstract}

\section{KEYWORDS}

Global Software Engineering, Offshore Development, SAFe, Scaled Agile Framework, Team Culture

\author{
ACM Reference format: \\ Alaukikdev Pandya, V. S. Mani, Ansuman Pattanayak. 2020. Expanding \\ the responsibility of an offshore team and sustainably increasing business \\ value using SAFe. In Proceedings of ICGSE '20: 15th IEEE/ACM \\ International Conference on Global Software Engineering. October 5--6, \\ 2020. Seoul, Republic of Korea, 5 pages. \\ https://doi.org/10.1145/3372787.3390441
}

\section{INTRODUCTION}

We have structured this experience report chronologically. This section describes the structure of the paper. Section 2 describes the offshore team's working context and the need for expansion. Section 3 outlines our approach for taking up additional responsibilities from early 2015 till 2016. Section 4 mentions the drivers that helped achieve high performance after taking on additional responsibilities from 2017 till 2019. Section 5 talks about the business need to sustainably increase the business value, which triggered the SAFe transformation. Section 6 covers the approach we adopted for transitioning from Scrum to SAFe during the first half of 2019. Section 7 presents the results achieved after the SAFe implementation up to the first quarter of 2020, and Section 8 and 9 respectively summarize the lessons learned and conclusions from the journey.

Permission to make digital or hard copies of all or part of this work for personal or classroom use is granted without fee provided that copies are not made or distributed for profit or commercial advantage and that copies bear this notice and the full citation on the first page. Copyrights for components of this work owned by others than ACM must be honored. Abstracting with credit is permitted. To copy otherwise, or republish, to post on servers or to redistribute to lists, requires

prior specific permission and/or a fee. Request permissions from ermissions@acm.org.

ICGSE '20, October 5-6, 2020, Seoul, Republic of Kore

C) 2020 Association for Computing Machinery.

ACM ISBN $978-1-4503-7093-6 / 20 / 05 \ldots \$ 15.00$

https://doi.org/10.1145/3372787.339044

\section{BACKGROUND}

Our organization is part of a global team that develops software for multiple product families of medical imaging systems. Quality is non-negotiable because of the missioncriticality of our products and the highly regulated nature of medical technology industry.

The global team is geographically distributed between India (Bangalore) and the U.S. Initially, the team in Bangalore was responsible for delivering multiple software modules of a single product line as an integral part of the global software organization. At that time, while the teams in both countries followed Scrum practices, the overall software delivery responsibility was not with the team in Bangalore. In 2015, it was decided to establish more global responsibilities in Bangalore to leverage the capabilities of the India team and optimize cost.

\section{APPROACH FOR EXPANSION}

The process of expanding and taking up additional responsibilities started in the middle of 2015 and had to be finished by the end of 2016. It was critical to ramp up the team quickly to realize the management objectives, especially business continuity. This section outlines the steps to ramp up quickly.

\subsection{Identifying the roles and candidates}

The roles to add in Bangalore were mainly identified based on 1) the long term vision of the organization, 2) the possibility of driving the responsibilities from Bangalore in accordance with regulations, and 3 ) the ability to ensure business continuity by quickly establishing the required competences.

The key roles identified included Global Project Manager, Line Manager, Product Owner, Test Lead, Project Architect, Quality Assurance (QA) Engineer, DevOps Engineer, and specialized software roles requiring expertise in new technologies.

For every identified role, the expected technical and behavioral competencies were defined along with the experts in partner organization to benefit from their prior experience. For all roles, we tried identifying suitable candidates within the team, which worked for most roles. For roles where we did not have internal candidates, we hired talent from outside.

\subsection{Skill gap analysis}

Once an individual joined for a role, the gap between the current and expected skill level was evaluated and represented as 
a spider chart. Based on this analysis, an individual-specific knowledge transfer and on-the-job learning plan were defined. The competency spider was tracked for individuals to assess their progress and carry out course correction as necessary.

\subsection{Knowledge transfer to drive responsibilities}

The knowledge transfer included following three phases:

3.3.1 Instructor-led training: Face-to-face training sessions on technical aspects, processes, and tools were conducted by an experienced onsite counterpart (mentor). Travel costs were budgeted. Impact of the training effort on the ongoing projects was addressed by carefully planning the required time for the activity and factoring in the reduced effort available for project delivery tasks. For new technology areas, we focused the training on the most critical topics identified based on the outlook.

3.3.2 Shadowing: New incumbent supported the mentor and learned on-the-job, actively contributed to the ongoing project and acquired knowhow. Readiness to take up the new role was assessed by reversing the shadowing process, where the new incumbent took the lead for identified tasks.

3.3.2 Driving: After considering the progress and the confidence of the individual and the view of the management, it was decided when a new incumbent would take up the complete responsibilities for the role.

The three-phase approach was effective in enabling the Bangalore team to take up additional responsibilities within the expected timeframe. But, it required considerable planning and coordination by the respective line manager along with the onsite organization. However, more time was needed for preparing software engineering roles in new technology areas, which required defining contingency measures.

\section{HIGH PERFORMANCE ACHIEVED}

In the three years following the expansion, the team in Bangalore focused on raising the bar on performance. During this time, the team independently delivered three product releases (typically annually) along with multiple patch releases (usually quarterly) successfully. The team also took up the complete software development responsibility for a new product, modules and technology areas. In the sub-sections below, we have outlined some of the steps taken to achieve high performance.

\subsection{Gaining the trust of stakeholders}

While offshoring, gaining trust of key stakeholders is vital and for this we followed the general orientation explained below.

4.1.1 Understanding stakeholders: Every individual identified their key stakeholders and their level of interest and influence on the role. For key roles, a stakeholder maps were created, which were used to define customized engagement plans. The map had four quadrants to place the stakeholders based on their interest and influence on the project, while also indicating their attitude.

4.1.2 Understanding stakeholders' expectations: It was essential to understand both the explicit (said) and implicit (unsaid) expectations of stakeholders, to fulfill them. We interviewed key stakeholders upfront to align the explicit expectations. However, the implicit expectations of stakeholders changed depending on the project phase and situation, so project managers had to recognize and realign with the changed implicit expectations of stakeholders.

4.1.3 Preparation for key decisions and status reporting: Regular meetings were scheduled to report the status to the executive management and decide the internal milestones. Therefore, project managers had to be prepared with accurate information and anticipate potential questions to ensure better decisions were made. Several times, mock presentations were conducted to improve the preparation.

4.1.4 Meeting expectations: Regularly seeking the feedback from the key stakeholders helped in identifying gaps and there by meeting the expectations and gaining trust. Despite this, the offshore team was at times unsuccessful in doing so.

\subsection{Tracking Progress}

To successfully steer project releases we defied, continuously tracked key performance indicators (KPIs) in the areas mentioned below to take timely corrective actions. Data collection and trending were largely automated using internal tools.

4.2.1 Project release: KPIs were derived for tracking the progress of scope, schedule, and cost. Some of these were: release and team burndown, feature completeness, project milestone readiness, cost variance.

4.2.2 Product quality: KPIs were defined to monitor product health. Some of the these were: feature 'done-ness', number of defects at team level, static code quality parameters, pass percentage of the daily integration tests, formal verification and validation goals and trending of non-functional requirements against the defined criteria.

4.2.3 Team performance: KPIs were defined to measure and improve team performance in terms of throughput and efficiency. Some of the these were: team's predictability, velocity, cycle time trend, defect fix rate.

\subsection{Improving maintainability}

Our data showed that the maintenance effort ranged from around half to three-fourths of the total project effort. Also, introducing new functionality was costlier when the architecture did not scale. Hence, it was necessary to improve the maintainability of the system to increase team's capacity to deliver desired new functionality without increasing the team size.

Improving maintainability required an initial investment towards aspects such as product architecture, test automation, technical debt management, product lifecycle management processes and practices. Over a three-year term, development of new functionality sped up.

\section{INCREASING BUSINESS VALUE}

About two years after taking up expanded software engineering responsibilities, the management focused on increasing business value with the same team size. Both onsite 
and India management teams worked together to identify the future business demands, success factors, and challenges in sustainably increasing business value, as elaborated below.

\subsection{Business demands}

Some of the identified business demands were:

5.1.1 Dynamically adapting to changed business priorities: Previously the scope was defined for a year, with each project team having separate commitments. This made it difficult to handle dynamic changes in priorities across the projects, which was essential to deliver the highest business value.

5.1.2 Sustainable predictability and high throughput: Despite the growing complexity and functionality of the products, it was necessary to ensure predictability and high throughput to realize the ambitious product and architectural roadmaps. Hence, teams had to be sustainably predictable with high throughput to achieve project milestones on time and within budget.

5.1.3 Team agility to work on different products: As different products had different architectures and domain-centric requirements, teams had to develop agility and competences to contribute across products. This was essential to achieve maximum business value with the same team size.

5.1.4 Increased transparency: While the development teams needed to know the changing business priorities, the management needed to see the progress made by the development team in realtime. Increased transparency both ways was essential to effectively adjust focus and plans, as needed.

5.1.5 Innovation focus: The project teams were continually focused on delivery, which led to burnouts. In this situation, the teams were unable to innovate and improve their efficiency. An innovation focus was essential to build the future product roadmap and enhance engagement of team members.

\subsection{Why SAFe?}

It was evident that the current project-focused setup with multiple Scrum teams would not be able to address these business demands. One of the main limiting factors was the project-specific backlog with dedicated teams working only to deliver their backlog for their yearly release cycle. This way of working enabled developing specialized competencies for specific products and components. But it created inflexibilities while working on different product domains and architectures. A more flexible yet equally efficient working model was required to realize the ambitious product and architectural roadmap. It also called for a different organization setup, one which enhances individual capabilities and enables teams to achieve the roadmap.

The management envisioned a better working model with $\mathrm{SAFe}$. It required moving from a project-centric organization at the project level to a product-centric one at the program level, enabled by a single product backlog that is prioritized across different products and project releases. To deliver more value with the available capacity, it was essential that all teams could pull tasks from the single backlog. For this, teams had to scale up their capabilities to work on different products.

\section{SAFe TRANSFORMATION}

After assessing the readiness to implement $\mathrm{SAFe}$ at a program level (Essential SAFe [1]), Agile Release Train (ART) launch was scheduled in the second quarter of 2019. It gave a four-month preparation window for the first Program Increment (PI) planning event. Achieving the minimum readiness to launch the ART required understanding and prioritizing the preparation steps with a fair degree of confidence. The following subsections outline the approach adopted for SAFe transformation.

\subsection{SAFe Transformation backlog}

The first step of the SAFe transformation was to identify the required activities and prioritize them in the backlog. These activities were systematically identified as mentioned below.

6.1.1 Workshop: A workshop to identify the risks and opportunities of moving to SAFe was organized with management team. Also, measures to mitigate risks and use the opportunities were defined. Further, the need for change in the process or toolchain to enable SAFe implementation was assessed.

6.1.2 Team agility assessment: Team agility was assessed using a survey [2], which helped in determining the current maturity of Scrum practices within the teams. Accordingly, a plan was defined for scaling agile with higher maturity.

6.1.3 Team culture assessment: A team dysfunction survey [3] was conducted. The results of this survey helped in identifying underlying dysfunctions within the teams, and in deriving specific measures to address them.

6.1.4 SAFe trainings: Based on their roles, every member of the team underwent the required SAFe training programs. Many training programs were conducted well before ART launch, so that trained team members were better prepared.

These activities were prioritized based on their importance to achieve the essential readiness ahead of the first PI planning. The $\mathrm{SAFe}$ transformation backlog was closely tracked during the preparation phase by the management team.

\subsection{Restructuring the organization}

A critical activity of the SAFe transformation backlog was restructuring the current organization to make it leaner and more efficient, while creating essential new roles needed for implementing SAFe successfully. Several role changes were identified and implemented before the ART launch. This was challenging as explained below:

6.2.1 Scale: Organization structure was fully revamped, which impacted the entire team.

6.2.2 Individual alignment: Restructuring was done mainly by mapping existing employees to new roles, which required the alignment of an individual for the role and the other way around.

6.2.3 Communication: Because the role-related decisions had a significant impact on an individual's career, and on the overall team performance, communication about role changes were carefully planned and executed in a specific sequence across individuals. In case an individual declined the primary role 
offered; alternate roles were defined. The communication of the remaining roles was accordingly adjusted for others.

6.2.4 Role redefinition with SAFe: Existing and newly identified roles had to be redefined, recognizing the clear separation of responsibilities to ensure a smoother transition. E.g., the Project Manager and Release Train Engineer (RTE) role had a significant overlap and were elaborated considering the SAFe guidelines and business focus of these roles. Some other roles like line manager, test lead, which were essential due to the business context, were also elaborated considering SAFe focus.

Some of the key organizational changes done: 1) one team one line manager: to achieve one team mindset and to breakdown the silos within the team, 2) introduction of the RTE role and reduction of project manager role due to that, 3) program architect and test lead roles identified, 4) internal reshuffling across Scrum teams to balance the experience and skills of the teams to work on any product, 5) A new system team was created to support SAFe recommended responsibilities.

Leadership buy-in and support made it possible to carry out such large-scale organization restructuring.

\subsection{Preparation for ART Launch}

There were several operational and strategic activities involved in preparing for the first PI planning event. Some of the key activities are mentioned below.

6.3.1 Organization readiness: To transition to SAFe, it was essential to have alignment of practices at the program level, which required changes to the team level Scrum practices. These practices included: harmonizing estimation approach, common definition of done, cadence and synchronization across the teams for iteration schedule, and so on. At this stage, an external SAFe consultant was engaged, who worked closely with the teams by sharing the required framework knowledge and helped fostering team dynamics for smoother transition to SAFe.

6.3.2 Content readiness: Achieving required levels of content readiness before PI planning was essential. It mainly involved elaborating the product and architectural backlog sufficiently and preparing teams for it. This helped define the high-level product and architectural roadmap with the flexibility of modifying it at the PI boundary. It also helped the team get the right focus and gain momentum in delivering the roadmap.

6.3.3 Facility readiness: Certain logistical arrangement such as a big-room, technical infrastructure is essential and must be available for the event. The first PI planning event was a success, and the ART launch was celebrated with a team dinner.

\section{RESULTS FROM SAFe IMPLEMENTATION}

The results after ten months of SAFe implementation follow:

\subsection{Transparency and visual management}

One of the explicit benefits of implementing SAFe was the enhanced transparency at all levels. E.g., Inspect and Adapt (I\&A) and PI planning events proved to be excellent platforms for sharing achievements, lessons learned, priorities, and dependencies. This increased transparency and enhanced the trust.

With SAFe, meetings were optimized. Focused meetings like Scrum of Scrums, Product Owner / Product Manager sync were introduced while the release-focused meetings were retained with revised intent. Emphasis was on keeping PI execution on-track while ensuring release objectives are not compromised.

Digital dashboards were developed to facilitate remote PI planning events and to track the PI execution progress in real-time at both program and team levels. This helped in making informed decisions to achieve desired predictability and throughput. These dashboards evolved overtime with stakeholder feedback. These dashboards were tracked in addition to the traditional project-level metrics, which helped in tracking both PI and Project progress. However, in some cases, physical dashboards were also used.

\subsection{Predictability and throughput delivered}

As mentioned in section 5.1.2, teams needed to be sustainably predictable with high throughput in order to achieve the committed PI objectives and long-term product roadmap. Throughput was measured in terms of the actual volume of work done by the team in each PI. It includes new features developed, defects fixed, number of internal project milestones achieved, and so on. Predictability and throughput, when seen together, gave a holistic view of performance the ART.

The key to achieving consistent high predictability and throughput is upfront planning and prioritization through the single product backlog. The program consistently achieved $90 \%$ predictability. As the output of multiple PI's make up a scope of yearly project release, the high predictability helped in determining the changes needed for achieving project releases.

\subsection{Results from the architectural roadmap}

While the team actively worked on delivering the architectural roadmap, the size of the overall code base and static code violations significantly reduced compared to previous releases. This also enabled further development of the product roadmap.

\subsection{Business demands met}

This section mentions the extent to which the specific business demands that triggered SAFe transformation were met.

7.4.1 Dynamically adapting to changed business priority largely met: It was possible to handle dynamic business priorities through PI planning. Teams worked on multiple projects in parallel by pulling the highest priority tasks from the single product backlog (refer section 5.2).

7.4.2 Sustainable predictability - largely met: The predictability was high which enabled planning project releases and achieving the committed product roadmap (refer section 7.2).

7.4.3 Sustainable high throughput - fairly met: Team throughput depends on the complexity of the backlog tasks, the prior experience of the team, and team capacity that varies across PIs. Despite these factors, team throughput was improved.

7.4.4 Team agility to work on different products - fairly met: Teams were able to pull many high priority tasks from the single 
product backlog. However, teams needed to develop more agility and competences to work on any product domain and architecture.

7.4.5 Increased transparency - largely met: Different measures (refer section 7.1) helped in significantly increasing visibility, focus, and triggering decisions to modify plans.

7.4.6 Innovation focus - fairly met: Teams were able to utilize 30 to $40 \%$ of Innovation and Planning (IP) iteration capacity in every PI for its intended purpose. This is far better than earlier, where hardly any capacity was available to innovate. Intended use of IP iteration was improving with management support.

\section{LESSONS LEARNED}

\subsection{Expanding responsibilities of an offshore team}

Time-bound expansion of responsibilities was critical for business continuity. The main lessons learned follow.

Filling the open positions with internal candidates, where possible, worked well for initiating knowledge transfer and taking up the responsibilities quickly. Identifying external candidates for key roles like Product Owner, required looking beyond the title and describing the main characteristic in the job description.

Effective and timely knowledge transfer depends on the detailed analysis of competency gap and planning accordingly. Knowledge transfer worked best when done face to face by sharing the know-how and experience, including do's and don'ts.

For all the new roles, the focus was first on building the required competence on-the-job (in domain and technology) to ensure business continuity rather than assessing improvement opportunities in the job, process and so on. The process of taking up the new responsibilities is considered complete only when it is established that the new incumbent in the role was able to gain the trust of global stakeholders.

\subsection{SAFe transformation}

We followed an experimental strategy which was adapted on the go, based on progress in terms of process, tools and more importantly the impact on the people. The lessons learned follow.

It is important to fix a date for PI planning in advance and adhere to it. This works as a guidance for backward planning. The time required to complete the most important preparation activities (refer section 6.3), guide in finalizing the date. This is the key to successful ART launch.

The organization restructuring needed both identifying individuals with the closest fit for the role and empathy to ensure that their career aspirations were being addressed. This wasn't straightforward and required thinking through alternatives upfront. Despite this, it is unreasonable to expect all employees will be satisfied immediately after the restructuring.

Several teams were unable to achieve the required level of readiness for PI planning due to committed internal project milestones and largely consumed IP iteration for backlog refinement. This reduced the effectiveness of the PI planning.

The big room-based PI planning and I\&A session are a must, as they improve social engagement, collaboration and learning.
Predictability and throughput typically increase gradually and depend on the ability of the ART to quickly adapt based on the feedback received from stakeholders and teams.

It is important to remain focused on long term project objectives for yearly releases while ensuring that the PI objectives are also met. At times this required the project managers to facilitate the change in priorities for the ongoing PI through RTE. It was challenging to handle dynamic prioritization conflicts.

More than anything, sustainable success of SAFe implementation depends on building the right team culture. We focused on strengthening trust and enhancing empowerment and accountability. Towards this we conducted team building exercises and numerous other interventions to strengthen these aspects.

\section{CONCLUSIONS}

For time bound expansion of responsibilities in the offshore team, it is essential to have the buy-in of the management team, individual roles and their onsite mentors. Another critical success factor is that individuals in the key roles need to be open to learn from constructive feedback.

$\mathrm{SAFe}$ is not a silver bullet, but rather a framework that must be evaluated for the given business context, considering the main demands of using it to achieve specific business outcomes. Also, there will likely be initial setbacks, even after implementing SAFe without any deviation from the recommended framework. These setbacks need to be assessed and improved upon quickly to achieve the desired business outcomes.

Even without using SAFe, the desired results could have been achieved, though it may have taken longer. Also, the results may not have been sustainable. SAFe helped in getting the results in more structured and sustainable manner by mandating implementation of certain aspects by the framework (e.g. PI planning, IP iteration and so on). A successful SAFe implementation is not a state but a journey. Like any other transformation of this scale, it takes time.

\section{REFERENCES}

[1] Scaled Agile Framework (SAFe) : https://www.scaledagileframework.com/

[2] Comparative Agility: www.comparativeagility.com

[3] “The Five Dysfunctions of a team" by Patrick Lencioni. ISBN: 9780787960759 